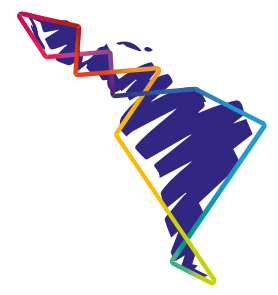

\title{
Jóvenes promotores de derechos: una experiencia de aprendizaje en el cantón de Garabito, Costa Rica
}

\section{Youths as promoters of rights: a learning experience in the Canton of Garabito, Costa Rica}

\author{
Julia Chan Jiménez ${ }^{1}$ \\ Nancy Sánchez Acuña² \\ María Eugenia Restrepo Salazar ${ }^{3}$
}

\begin{abstract}
Resumen
Este documento busca compartir el proceso de construcción de la propuesta "Jóvenes promotores de derechos", basada en una experiencia de aprendizaje con jóvenes entre 13 y 22 años de la comunidad de Garabito, Pacífico Central de Costa Rica, durante los años 2016 y 2017. Surge de un proceso de investigación, sensibilización y formación en derechos humanos y cultura de paz, en el marco del proyecto "Ciudades culturales: UNA alianza para el futuro", ejecutado por académicas de las vicerrectorías de Extensión y de Docencia de la Universidad Nacional de Costa Rica (UNA), en asocio con el Centro Cívico para la Paz (CCP) de Garabito y el Colegio Técnico Profesional de Jacó (CTP), así como con la participación de estudiantes de grado y posgrado de la UNA y de la Universidad Estatal a Distancia (UNED). El proceso realizado con
\end{abstract}

1 Socióloga de la Universidad de Costa Rica (UCR). Máster en Gerencia de Proyectos (ICAP). Asesora Académica del Proceso de Gestión Proyectos en la Vicerrectoría de Docencia de la UNA. Representante de la UNA, Costa Rica, en la Subcomisión de proyectos de docencia del Consejo Nacional de Rectores (CONARE), y en otras comisiones de carácter interinstitucional. Participa en el proyecto "Ciudades Culturales: una alianza para el futuro" del Centro de Estudios Generales de la UNA, Costa Rica. Docente de la Maestría en Evaluación de Programas y Proyectos de Desarrollo de la UCR, Costa Rica.

2 Bióloga de la Universidad Nacional de Costa Rica (UNA). Máster en Desarrollo Comunitario Sustentable UNA. Coordinadora de la Unidad Vínculo Universidad, Sociedad y Estado y asesora en Gestión de proyectos en la Vicerrectoría de Extensión de la UNA. Coordina el proyecto "Ciudades Culturales: una alianza para el futuro" del Centro de Estudios Generales de la UNA, Costa Rica. Docente en el Centro de Estudios Generales de la UNA, Costa Rica.

3 Bióloga de la Universidad Nacional de Costa Rica (UNA). Magíster en Derechos Humanos de la Universidad Estatal a Distancia (UNED). Asesora Académica de Proyectos de Extensión Universitaria en la Universidad Nacional, Costa Rica. Editora en jefe de la Revista Universidad en Diálogo de la Vicerrectoría de Extensión (UNA). Integrante de la Subcomisión de Capacitación Interuniversitaria del Consejo Nacional de Rectores (CONARE). Docente de la Sede Interuniversitaria de Alajuela, Costa Rica. 
apoyo de herramientas ludopedagógicas constó de 3 fases; la primera es de tipo diagnóstico, en la cual se exploran las percepciones de las personas jóvenes acerca de los problemas de su comunidad, la violencia en sus diferentes expresiones y la vivencia de los derechos humanos. La segunda etapa corresponde al fortalecimiento de capacidades en temas de liderazgo, derechos humanos y cultura de paz tanto a jóvenes del CCP como del CTP. La tercera fase se dedica al pilotaje de la propuesta únicamente con jóvenes de décimo nivel del CTP. Como resultado, se tiene una proposición participativa de sensibilización y educación, dirigida a jóvenes con el objetivo de que sean promotores de derechos en su centro educativo y su comunidad.

Palabras clave: cultura de paz, derechos humanos, juventud, ludopedagogía, propuesta metodológica.

\section{Summary}

This document seeks to share the process by which the "Jóvenes Promotores de Derechos" ("Youth Rights Promoters") proposal was constructed based on a learning experience carried out with young people between ages 13 to 22 from the community of Garabito in the Central Pacific region of Costa Rica during the years 2016 and 2017. The subject matter arises from a process of investigation, sensitization and training in Human Rights and Culture of Peace within the framework of the Proyecto Ciudades culturales: UNA alianza para el futuro (Cultural Cities Project: UNA alliance for the future), executed by academic personnel of the Vice-Rectories of Extension and of Education (Vicerectorías de Extensión y de Docencia) of the Universidad Nacional of Costa Rica (UNA) (National University of Costa Rica) in partnership with the City of Garabito's Centro Cívico para la Paz (CCP) (Civic Center for Peace) and the City of Jaco's Colegio Técnico Profesional (CTP) (Professional Technical High School), with participation of undergraduate and graduate students from UNA and Universidad Estatal a Distancia (UNED) (State Distance-Learning University). The process carried out with the support of ludopedagogical tools consisted of three phases; the first, of diagnostic nature, explored young people's perceptions about the problems in their community, violence in its different expressions and the experience of Human Rights. The second phase corresponds to the strengthening of capacities in issues of leadership, Human Rights and Culture of Peace for young people from both CCP and CTP. The third phase corresponds to a proposal pilot provided only to $10^{\text {th }}$ grade youths from CTP. As a result, we arrive at a participatory proposal of sensitization and education aimed at young people with the objective forming them as promoters of rights in their educational centers and communities.

Keywords: Culture of Peace; Human Rights; Youth; Ludopedagogy; Methodological proposal. 


\section{Contextualización de la experiencia}

Los derechos humanos son sus derechos. Tómenlos. Defiéndanlos. Promuévanlos. Entiéndanlos e insistan en ellos. Nútranlos y enriquézcanlos. . . Son lo mejor de nosotros. Denles vida. Martin Luther King

Esta experiencia de aprendizaje en derechos humanos y cultura de paz surge en el marco del proyecto "Ciudades Culturales: UNA alianza para el futuro", con vigencia 2016-2020, ejecutado en el cantón de Garabito, provincia de Puntarenas, y dirigido por académicas de las vicerrectorías de Extensión y de Docencia de la UNA. Este busca contribuir con la generación de acciones que propicien el desarrollo de ciudades fundamentadas en los derechos humanos, la inclusión y el respeto por lo diverso, en las cuales la niñez y la adolescencia puedan aportar desde su visión para la generación de nuevos espacios donde comprender y ejercer sus derechos.

La estrategia de gestión del proyecto se basa en tejer redes de trabajo con actores internos de la UNA, al igual que con actores externos institucionales y de base comunitaria. Se articula con unidades académicas, programas, proyectos y cursos de la UNA, para que el estudiantado realice prácticas y trabajos finales de graduación relacionados con los objetivos del proyecto. De este modo, se integraron a esta experiencia estudiantes de la Escuela de Relaciones Internacionales, la Escuela de Psicología y la Federación de Estudiantes (FEUNA). Se contó, además, con una tesiaria de la Maestría en Derechos Humanos de la Universidad Estatal a Distancia (UNED).

A lo externo, la articulación se realizó con el Centro Cívico para la Paz (CCP) de Garabito, llamado "Epicentro", y el Colegio Técnico Profesional de Jacó, del Ministerio de Educación Pública (MEP), que, como veremos más adelante, se involucró en la segunda y tercera etapas de esta experiencia.

Los CCP forman parte de la iniciativa de gobierno titulada "Programa para la prevención de la violencia y la promoción de la inclusión social" del Ministerio de Justicia y Paz. Existen 7 CCP, 1 en cada provincia del país, en cantones seleccionados por su alto índice de violencia y, según lo planteado por Grillo (2014), estos son un espacio físico con sitios para el deporte y la recreación, la música, la tecnología, biblioteca, salas de formación y otros servicios especialmente diseñados para jóvenes menores de 18 años. Su modelo base de atención se sustenta en el enfoque de derechos, género, diversidad e interculturalidad y su oferta de información y formación se agrupa en 3 grandes ejes temáticos: 1 . autocuido y cuido, referido fundamentalmente a temas de violencia en sus diferentes manifestaciones; 
2. promoción de la inclusión social, agrupando tópicos de educación y empleo, y 3. convivencia y cultura de paz, destacando asuntos de democracia, participación ciudadana y resolución de conflictos.

Los ejes temáticos de mayor afinidad con el proyecto Ciudades Culturales son el 1 y el 3, los cuales se han trabajado de forma conjunta, dado que los temas de violencia se relacionan de modo directamente inverso con la convivencia pacífica y el respeto por los derechos humanos.

Tal afinidad de objetivos y temáticas nos llevó al cantón de Garabito a construir y desarrollar esta experiencia de aprendizaje con jóvenes de la localidad, durante los años 2016 y 2017, y que hoy compartimos en esta comunicación.

El cantón de Garabito es el número 11 de la provincia de Puntarenas y está dividido en 2 distritos: Jacó y Tárcoles. El primero se conoce por tener mayor concentración poblacional, actividad económica, cultural y social del cantón. Es un importante polo de inversión extranjera, especialmente en cuanto a construcción de hoteles de playa, centrando su principal actividad productiva en el turismo, que involucra otras ramas trascendentales como la hotelería, los restaurantes, la construcción y el comercio. Esto según la Municipalidad de Garabito (2014), en su Diagnóstico Cantonal.

En contraposición, encontramos una comunidad caracterizada por la presencia de drogas, narcotráfico, prostitución, tráfico de personas, migración y violencia. Chan, Sánchez y Víquez (2017), en un estudio de percepción de la violencia con niños y jóvenes del cantón, plantean que

... las formas de violencia más frecuentes son el maltrato físico y verbal, que la calle es considerada por esta población como altamente peligrosa y violenta para todas las personas, identificando la represión policial como una forma más de violencia en la calle (p. 19).

En este contexto, se realizó la mencionada experiencia de aprendizaje con personas jóvenes, con el objetivo de que fueran promotoras de derechos en su centro educativo y su comunidad. 


\section{Referente teórico metodológico del proceso de aprendizaje}

El cerebro no es un vaso por llenar, sino una lámpara por encender.

Catón

Este proceso de aprendizaje se encuentra sustentado en un grupo de conceptos que, además de dar sentido, orientan el accionar hacia una de las problemáticas definidas como prioritarias, como es la falta de conocimiento en el tema de los derechos humanos en la población juvenil.

En esta línea, se plantean una serie de conceptos y categorías que dan coherencia y consistencia a esta construcción conjunta con el grupo de jóvenes en Jacó, partiendo de la Declaración Universal de los Derechos Humanos (1948), articulo 2 , en el que se define como derechos humanos "aquellos que son inherentes al ser humano" y a los cuales "toda persona tiene todos los derechos y libertades, sin distinción alguna de raza, color, sexo, idioma, religión, opinión política o de cualquier otra índole, origen nacional o social, posición económica, nacimiento o cualquier otra condición". Esta misma declaratoria enfatiza en su preámbulo que

todos los pueblos y naciones deben esforzarse, a fin de que tanto las personas como las instituciones, inspirándose constantemente en ella, promuevan mediante la enseñanza y la educación el respeto a estos derechos y libertades, y aseguren a través de medidas progresivas de carácter nacional e internacional su reconocimiento y aplicación universales y efectivos.

Ese llamamiento es atendido con esta propuesta y con lo solicitado en la Conferencia Internacional de Educación de la UNESCO (2008), de educar para la convivencia democrática y pacífica, la cual "aspira a desarrollar en los estudiantes una disposición, una simpatía y un sentido hacia esta visión de vida, y además, debe entrenarlos para desarrollar en ellos las habilidades que se requieren para la vida en común”. (p. 14) Al respecto, Barquero-Brenes (2014) señala que:

La promoción de la convivencia no solo es un factor de bienestar para las personas, sino la base desde la que se construyen la ciudadanía, el capital social, la calidad de un país en el futuro y también la posibilidad de entendimiento entre los pueblos. Aprender a convivir incide de manera directa en el combate de formas violentas de relación y, por lo tanto, en la construcción de una cultura de paz (p. 2). 
Para Tuvilla (citado por Barquero-Brenes, 2014, p. 5), la cultura de paz representa

un gran desafío para la humanidad en un siglo lleno de grandes incertidumbres y también de grandes esperanzas, y la comprende como: Un concepto síntesis que encuentra en los derechos humanos su esencia básica y que se define como el conjunto de valores, actitudes, tradiciones, comportamientos y estilos de vida que inspiran una forma constructiva y creativa de relacionarnos para alcanzar la armonía del ser humano consigo mismo, con los demás y con la naturaleza (p. 5).

Por su lado, Barahona (2008) opina que cultura de paz es

... fundamentalmente el intento de la creación de una cultura donde impere la cooperación y la armonía, que venga a sustituir la cultura de choque y competencia en la cual vivimos actualmente. La Educación para la Paz, no la vemos como una materia o una disciplina más que hay que agregar a un plan de estudios; todo lo contrario: educar para la paz significa un contenido y un proceso global de la sociedad, a través de los cuales las personas y los grupos sociales aprendan a desarrollar conscientemente... la totalidad de sus capacidades, actitudes, aptitudes y conocimientos (p. 37).

Los elementos que constituyen la cultura de paz son fundamentales para establecer la justicia y el bienestar social, sin embargo, se deben consolidar acciones de compromiso para su logro, mediante el respeto de los derechos humanos, dejando de lado las desigualdades y fortaleciendo la educación, la transformación social e institucional de la sociedad civil. Al respecto, Garrido (2017) menciona que

... la promoción de la paz no es una tarea exclusiva de los estados, ya que la sociedad civil también está llamada a contribuir activamente en el reto de neutralizar la violencia, de modo que se promueva y se practique la paz a nivel comunitario, vecinal, escolar, etcétera (párr. 3).

El proceso para la consolidación de una cultura de paz en el nivel de una comunidad conlleva la participación de diversos actores, desde el núcleo familiar hasta representantes de instituciones públicas y privadas que se aboquen a la resolución pacífica de conflictos y al fortalecimiento de los derechos humanos fundamentales, como acciones principales. Por otra parte, Garrido (2017) plantea que

La paz es mucho más que la ausencia de guerra, requiere sociedades que vivan en equidad, libertad, igualdad y solidaridad, entre otros valores; es por ello que la construcción de paz es un proceso que comienza con la promoción de la cultura de paz, para que a través de la educación se instauren todos esos valores asociados que permitan alcanzar la meta (párr. 5 y 6). 
En lo que respecta a cómo percibe y vive los derechos y la cultura de paz la persona joven-adolescente, el Fondo de las Naciones Unidas para la Infancia (2006) contextualiza lo siguiente:

En la adolescencia aparecen aspectos como el desarrollo de la identidad, el sentido de autonomía, la capacidad para cuestionarse el sistema de referencia, la interacción con la sociedad. Es un período clave en el desarrollo humano porque se estructuran y se reestructuran las relaciones con uno mismo y con el mundo. Por ello, la adolescencia puede entenderse como el resultado de una construcción histórica y de un proceso social (p. 9).

De ahí que el entorno familiar, el círculo social de amigos y demás espacios de interacción constituyen escenarios muy importantes para el desempeño de las personas adolescentes en la sociedad y las diferentes formas de integrar lo social, lo económico y lo cultural a su desarrollo. Lamentablemente en estos espacios de interacción, la violencia es un factor que cada vez se hace más frecuente, no solo en el plano familiar, sino también en el nivel de instancias educativas, afectando su derecho a vivir y crecer en contextos de paz, a aprender y a expresarse libremente (Trucco e Inostroza, 2017).

Pinheiro (citado por Trucco e Inostroza, 2017) señala cómo en los últimos años el tema de la violencia en centros educativos ha sido de los más estudiados, dada la cantidad de tiempo que invierte y la convivencia que experimenta la población infantil y adolescente en estos lugares (p. 15), con datos que muestran altos niveles de agresividad, principalmente en la escuela y en la comunidad.

Ante este escenario y con el llamado de educar para la paz, la ludopedagogía, como herramienta metodológica, ayudó a este propósito por las diversas formas a través de las cuales se puede llegar al conocimiento. Melissa Melino (citada por Fulladosa, 2015) define la disciplina como aquella "...metodología que permite instalar en un contexto grupal la posibilidad de jugar con otros, para desde ese espacio de juego, construir una mirada crítica y transformadora de la realidad que vivimos" (p. 9). Mario Pyñeiro, también citado por esta autora, continúa ampliando lo que es y significa la ludopedagogía y la plantea como

... el camino de buscar formas de entender la realidad, a nivel individual y las relaciones entre las personas, de ampliar las posibilidades de abordar los procesos de construcción de conocimiento, con la integración de lo corporal, las sensaciones y sentimientos, así como la racionalidad, con el objetivo de contribuir a la transformación de la realidad en un sentido liberador, humanizante (p. 10). 
Fulladosa (2015) recalca lo anterior, diciendo que es lo que "nos permite aproximarnos desde la razón y los sentidos a la realidad social, con el fin de conocer y comprender su complejidad, esta que se manifiesta en lo azaroso, en lo incierto, en la alegría, en lo pasional, en el conflicto, en el ocio, es decir allí, en todo lo real que tiene y que se experimenta en el cotidiano" (p. 10).

Rickly y Ara del Amo (2011) mencionan que "el juego es una actividad libremente elegida, que otorga el permiso de transgredir normas de vida internas y externas; un satisfactor sinérgico de necesidades humanas, que abarca la dimensión individual y colectiva y tiene incidencia en el ámbito social, cultural y político" (p. 1).

Cerdas (2013) señala que

... los juegos cooperativos generan oportunidades para el desarrollo de las relaciones humanas, las responsabilidades individuales y colectivas, la creatividad, la resolución alternativa de conflictos, la comunicación, el trabajo en equipo, el desarrollo y fortalecimiento de habilidades que coadyuvan al desarrollo de la personalidad.... es un espacio poco común, de disfrute, libertad, participación y cooperación. Además, permiten aflorar interacciones socioafectivas, esto implica más que el desarrollo intelectual, de conocimientos y técnicas, sino de relaciones afectivas y solidarias que permiten a los/as participantes ponerse en los zapatos de los otros, la comprensión mutua, la empatía y generar un sentido de responsabilidad y de identificación con las necesidades. Las experiencias con los juegos cooperativos reflejan la necesidad de fortalecer el trabajo en equipo, generar espacios no tradicionales para formular nuevas propuestas, así como para recreación y disfrute individual y grupal (p. 121).

El educar para la paz, por tanto, ha de ser un esfuerzo por contrarrestar la cultura de la violencia y consolidar una nueva manera de ver, entender y vivir el mundo. En esta propuesta educativa de Jóvenes promotores de derechos, concebimos a los promotores como aquellos que contribuyen a dar impulso a los valores, las actitudes y los comportamientos de una cultura de paz. Son los llamados a guiar, acompañar y motivar el proceso de aprendizaje, en los temas de derechos humanos y cultura de paz. Este pensamiento concuerda con lo definido por la UNESCO (1997) para un promotor de la paz, como el que

...interioriza e institucionaliza los valores, posturas, conocimientos y procederes ligados a la cultura de paz ... ayudando a la gente a aprender los entresijos de la mediación y de la transformación de conflictos (p. 4). Los promotores de la paz siguen un proceso de aprendizaje que les permite añadir los métodos y principios de una cultura de paz a la formación y experiencias que hayan adquirido en su trayectoria 
profesional, suma de aptitudes que luego podrán transmitir a la gente con la que trabajan, transformando así el conflicto en cooperación (p. 5).

El enfoque de juventudes permite a los jóvenes, sean promotores o bien participantes, expresarse libremente, sabiéndose sujetos de derechos, actores estratégicos del desarrollo y personas capaces de ejercer responsablemente los derechos y libertades, tal y como lo dicta la Convención Iberoamericana de Derechos de los Jóvenes. Así, se miran no como una población vulnerable, sino como personas capaces de brindar aportes importantes desde sus capacidades, donde los estereotipos de edad, raza, religión o género no caben ni limitan su accionar. En los artículos 2 y 3 de esta convención, se

Reconoce el derecho de todos los jóvenes a gozar y disfrutar de todos los derechos humanos, y se comprometen a respetar y garantizar a los jóvenes el pleno disfrute y ejercicio de sus derechos civiles, políticos, económicos, sociales, y culturales. Se comprometen a formular políticas y proponer programas que alienten y mantengan de modo permanente la contribución y el compromiso de los jóvenes con una cultura de paz y el respeto a los derechos humanos y a la difusión de los valores de la tolerancia y la justicia (p. 3).

Luego de transitar el referente teórico de la experiencia, se presenta las distintas fases del proceso de aprendizaje realizado con los jóvenes de Jacó.

\section{La experiencia de aprendizaje con la juventud de Garabito}

El camino recorrido para la construcción de la propuesta educativa en el tema de derechos humanos y cultura de paz, por razones metodológicas, se dividió en 3 fases, sin embargo, hubo un proceso continuo no lineal de aprendizaje, en el cual estas se entrelazan entre sí y se potencian mutuamente. La primera fase llamada "Valoración de la problemática" es diagnóstica tanto de las condiciones locales como de la percepción de la juventud acerca de la violencia; la segunda, denominada "Fortalecimiento de capacidades", trata temas de liderazgo, derechos humanos y cultura de paz con los jóvenes, y la tercera, "Promotores de paz", es una propuesta piloto de intervención para la sensibilización en derechos humanos y cultura de paz, realizada con jóvenes escolarizados.

\section{Valoración de la problemática}

Con la valoración de la problemática, en sí misma, un diagnóstico social de las percepciones de las personas jóvenes acerca de su comunidad, pretendíamos 
conocer el imaginario y la realidad de los derechos humanos, así como marcar una ruta de contribución de la UNA en esta materia.

Nuestro primer acercamiento con las personas jóvenes de Garabito se da en el marco de la articulación con el Centro Cívico para la Paz (epicentro) y la Escuela de Relaciones Internacionales de la UNA, a partir de la realización de la Práctica Profesional Supervisada: "Alianza para la Construcción de una Cultura de Paz", de febrero a noviembre del 2016. Se sumó a esta experiencia, en el segundo semestre del 2016, la Escuela de Psicología, con la Práctica Profesional Supervisada: "Caracterización de la ciudad de Jacó y formación de jóvenes líderes en el Centro Cívico para la Paz de Garabito". Ambas prácticas fueron realizadas por 2 estudiantes que aspiraban al título de bachillerato en sus respectivas carreras. Se integra también 1 estudiante tesiaria de la Maestría en Derechos Humanos de la Universidad Estatal a Distancia (UNED).

En el cuadro 1, se presenta un resumen de las actividades realizadas, siempre con una metodología lúdica y participativa, tal como se describió en el segundo apartado de esta comunicación. Hubo un primer bloque de actividades, relacionado con talleres de educación para la paz, en el que cada semana se analizaba el conocimiento, la vivencia y la percepción de un derecho. El segundo bloque se relaciona con actividades más colectivas y de mapeo comunitario.

Se contó con la participación de 6 mujeres y 4 hombres en un rango de edad entre los 13 y los 22 años, con condición socioeconómica baja o muy baja, en proceso de conclusión del ciclo diversificado, bajo la modalidad de Colegio Académico Nocturno, con trabajos diurnos ocasionales. Ellos asistían al curso de inglés que se impartía en el CCP, como parte de la oferta programática del centro. A finales del año, en el marco de la celebración del "Día Internacional de la Paz", se sumaron otras personas jóvenes del CCP y de la comunidad.

\section{Cuadro 1. Actividades realizadas en la fase 1}

\begin{tabular}{|c|c|c|c|}
\hline Fecha & Tema & Objetivos & Actividades \\
\hline Abril & $\begin{array}{l}\text { Introducción al } \\
\text { tema de DDHH }\end{array}$ & $\begin{array}{l}\text { Conocernos entre } \\
\text { nosotros. } \\
\text { Conocer el marco } \\
\text { general de los } \\
\text { derechos humanos. }\end{array}$ & $\begin{array}{l}\text { Presentación en parejas. Una persona } \\
\text { del grupo realiza un dibujo, oculto } \\
\text { para los demás Luego, intenta dar } \\
\text { instrucciones al resto, para que } \\
\text { lo reproduzcan en sus papeles. Al } \\
\text { finalizar, se compara el original con las } \\
\text { reproducciones realizadas. Reflexionar } \\
\text { acerca de hablar, escuchar e interpretar. }\end{array}$ \\
\hline
\end{tabular}




\begin{tabular}{|c|c|c|c|}
\hline Fecha & Tema & Objetivos & Actividades \\
\hline Mayo & $\begin{array}{l}\text { Derecho a } \\
\text { la libertad } \\
\text { de opinión y } \\
\text { expresión }\end{array}$ & $\begin{array}{l}\text { Identificar la } \\
\text { importancia } \\
\text { del respeto a la } \\
\text { diversidad de } \\
\text { opiniones como } \\
\text { punto de partida de } \\
\text { la experiencia. } \\
\end{array}$ & $\begin{array}{l}\text { Pequeño conversatorio sobre la } \\
\text { importancia de expresar nuestros } \\
\text { pensamientos y opiniones libremente. } \\
\text { Se elabora un papelógrafo con las ideas } \\
\text { recabadas. } \\
\text { Video-foro "Youth for Human Rights". }\end{array}$ \\
\hline \multirow[t]{4}{*}{ Junio } & $\begin{array}{l}\text { Derecho a la } \\
\text { vida }\end{array}$ & $\begin{array}{l}\text { Identificar la vida } \\
\text { como principal } \\
\text { derecho de toda } \\
\text { persona. }\end{array}$ & Conversatorio sobre historias de vida. \\
\hline & $\begin{array}{l}\text { Derecho a la no } \\
\text { discriminación }\end{array}$ & $\begin{array}{l}\text { Determinar las } \\
\text { principales causas } \\
\text { de discriminación } \\
\text { en Jacó. }\end{array}$ & $\begin{array}{l}\text { Los participantes, en un papelógrafo, } \\
\text { destacan cuáles son las principales } \\
\text { causas de discriminación que se viven } \\
\text { en la comunidad y cómo pueden ser } \\
\text { abordadas. } \\
\text { Taller de teatro y expresión cultural. }\end{array}$ \\
\hline & $\begin{array}{l}\text { Género y } \\
\text { diversidad }\end{array}$ & $\begin{array}{l}\text { Comprender el } \\
\text { concepto de género } \\
\text { en el marco de los } \\
\text { estereotipos sociales } \\
\text { y la diversidad. }\end{array}$ & $\begin{array}{l}\text { Siluetas: Dibujar } 2 \text { siluetas, una de un } \\
\text { hombre y otra de una mujer, en las } \\
\text { cuales los participantes coloquen sus } \\
\text { pensamientos acerca del género opuesto. } \\
\text { Cineforo "Sufragistas". } \\
\text { Creación de una propuesta grupal para } \\
\text { instaurar la equidad de género en la } \\
\text { comunidad }\end{array}$ \\
\hline & $\begin{array}{l}\text { Derecho a un } \\
\text { medio ambiente } \\
\text { sano y limpio }\end{array}$ & $\begin{array}{l}\text { Crear conciencia } \\
\text { sobre el cuido del } \\
\text { medio ambiente. }\end{array}$ & $\begin{array}{l}\text { Taller sobre la reutilización de botellas de } \\
\text { plástico y de aluminio para la creación } \\
\text { de artefactos novedosos y útiles en el } \\
\text { día tras día para el grupo. Estos les son } \\
\text { solicitados con anterioridad al taller. }\end{array}$ \\
\hline Julio & $\begin{array}{l}\text { Derecho a la } \\
\text { privacidad }\end{array}$ & $\begin{array}{l}\text { Comprender } \\
\text { los procesos de } \\
\text { globalización y } \\
\text { cómo estos pueden } \\
\text { afectar el derecho a } \\
\text { la privacidad. } \\
\end{array}$ & $\begin{array}{l}\text { Taller sobre el uso adecuado de redes } \\
\text { sociales. }\end{array}$ \\
\hline
\end{tabular}




\begin{tabular}{|c|c|c|c|}
\hline Fecha & Tema & Objetivos & Actividades \\
\hline Agosto & $\begin{array}{l}\text { Derecho } \\
\text { al ocio y al } \\
\text { esparcimiento }\end{array}$ & $\begin{array}{l}\text { Analizar el ocio y } \\
\text { el esparcimiento } \\
\text { como derechos } \\
\text { de cada persona } \\
\text { para su realización } \\
\text { personal. }\end{array}$ & $\begin{array}{l}\text { Rally de trabajo en equipo. Se elaboran } \\
4 \text { estaciones con duración de } 20 \text { min } \\
\text { cada una. El grupo se divide en } 4 \\
\text { subgrupos. Estación 1: A cada uno } \\
\text { se le da una escoba. Se colocan } 2 \\
\text { mesas volteadas a cada lado del salón, } \\
\text { a manera de portería. Los jugadores } \\
\text { deben barrer una pelota pequeña de } \\
\text { hule o de plástico a la portería del } \\
\text { equipo contrario. Como premio, se } \\
\text { obtiene una pieza de rompecabezas. } \\
\text { Estación 2: Se juega como el fútbol, } \\
\text { solo que los equipos se dividen en } \\
\text { pares. Luego se atan un pie al de su } \\
\text { pareja, por lo que deben trabajar } \\
\text { en equipo para pegarle a la pelota y } \\
\text { caminar. Cuentan los goles cuando } \\
\text { se hacen con el pie, que es más difícil } \\
\text { de manejar (por ej.: si se es derecho, } \\
\text { se debe patear con el pie izquierdo y } \\
\text { viceversa). Como premio, se obtiene } \\
\text { una pieza de rompecabezas. Estación } 3: \\
\text { Divide al grupo en } 2 \text { equipos separados } \\
\text { por una línea al centro. El objetivo } \\
\text { es que los integrantes de cada equipo } \\
\text { crucen al área enemiga y arranquen la } \\
\text { bandera de los contrarios. Ellos deben } \\
\text { evitar ser tocados por los jugadores } \\
\text { del otro equipo. Si alguien es tocado, } \\
\text { se queda congelado hasta que algún } \\
\text { compañero de su equipo lo toque y } \\
\text { pueda regresar a su zona. El equipo que } \\
\text { captura la bandera y regresa a salvo a } \\
\text { su lado gana. Como premio, se obtiene } \\
\text { una pieza de rompecabezas. Estación } \\
4: \text { Coloca una red baja. Utilizando una } \\
\text { pelota inflable grande, todos juegan } \\
\text { voleibol; nada más que deben estar } \\
\text { sentados en el suelo. Como premio, se } \\
\text { obtiene una pieza de rompecabezas. El } \\
\text { premio final es el rompecabezas armado } \\
\text { con palabras clave. }\end{array}$ \\
\hline
\end{tabular}




\begin{tabular}{|c|c|c|c|}
\hline Fecha & Tema & Objetivos & Actividades \\
\hline & $\begin{array}{l}\text { Derecho a la } \\
\text { educación }\end{array}$ & $\begin{array}{l}\text { Analizar la } \\
\text { importancia de } \\
\text { la formación } \\
\text { profesional. }\end{array}$ & $\begin{array}{l}\text { Mediante un taller sobre el uso de } \\
\text { Word, los participantes deben realizar } \\
\text { un corto ensayo sobre la importancia } \\
\text { de la educación, o bien acerca de algún } \\
\text { tema relacionado. }\end{array}$ \\
\hline \multirow[t]{2}{*}{ Sept. } & $\begin{array}{l}\text { Derecho a la } \\
\text { educación }\end{array}$ & $\begin{array}{l}\text { Analizar la } \\
\text { importancia de } \\
\text { la formación } \\
\text { profesional } \\
\text { (continuación del } \\
\text { taller anterior). }\end{array}$ & $\begin{array}{l}\text { Mediante un taller sobre el uso de } \\
\text { Power Point, lo estudiantes elaboran } \\
\text { una breve presentación acerca de las } \\
\text { ideas expuestas en el ensayo elaborado } \\
\text { en el taller anterior. }\end{array}$ \\
\hline & $\begin{array}{l}\text { Derechos } \\
\text { políticos: } \\
\text { participación } \\
\text { ciudadana }\end{array}$ & $\begin{array}{l}\text { Destacar la } \\
\text { importancia de } \\
\text { la participación } \\
\text { ciudadana. }\end{array}$ & $\begin{array}{l}\text { Términos y sus conceptos. Se divide } \\
\text { el grupo en parejas o subgrupos, se } \\
\text { colocan conceptos relacionados con } \\
\text { la participación ciudadana, se leen } \\
\text { aleatoriamente estos conceptos y los } \\
\text { subgrupos deben escoger cuál es el } \\
\text { término correspondiente. } \\
\text { Actividad de "si" o "no". Se colocan } 2 \\
\text { carteles en la pizarra: uno de "sí" y otro } \\
\text { de "no", se leen algunas afirmaciones } \\
\text { y los participantes deben colocarse del } \\
\text { lado del "si" o del "no", dependiendo } \\
\text { de lo que opinen. } \\
\text { Elaboración de un "programa de acción } \\
\text { ciudadana". }\end{array}$ \\
\hline \multirow[t]{2}{*}{ Octubre } & $\begin{array}{l}\text { Feria de } \\
\text { Derechos } \\
\text { Humanos }\end{array}$ & $\begin{array}{l}\text { Dar a conocer a la } \\
\text { comunidad el tema } \\
\text { de los DDHH. }\end{array}$ & $\begin{array}{l}\text { Elaboración de la manta "Conoce más } \\
\text { de tus derechos y plasma tu huella", } \\
\text { en la cual se colocan mensajes o frases } \\
\text { sobre los derechos. }\end{array}$ \\
\hline & $\begin{array}{l}\text { Mapeo } \\
\text { psicosocial } \\
\text { comunitario }\end{array}$ & $\begin{array}{l}\text { Conocer las } \\
\text { representaciones } \\
\text { que se tienen sobre } \\
\text { la comunidad. } \\
\text { Hacer inventario } \\
\text { de los recursos } \\
\text { existentes en la } \\
\text { comunidad, su uso } \\
\text { y estado. }\end{array}$ & $\begin{array}{l}\text { Es un soporte gráfico y visual, mediante } \\
\text { el cual se visibilizan las problemáticas } \\
\text { más urgentes del territorio, } \\
\text { identificando a los responsables, } \\
\text { reflexionando sobre conexiones } \\
\text { con otras temáticas y señalando las } \\
\text { consecuencias. } \\
\text { Mapeo de actores: ¿Cuáles } \\
\text { organizaciones hay en mi comunidad y } \\
\text { cómo acudir a ellas? } \\
\text { Análisis FODA de la comunidad. }\end{array}$ \\
\hline
\end{tabular}




\begin{tabular}{|c|c|c|c|}
\hline Fecha & Tema & Objetivos & Actividades \\
\hline & $\begin{array}{l}\text { Conflictos y } \\
\text { mediación }\end{array}$ & $\begin{array}{l}\text { Identificar } \\
\text { situaciones de } \\
\text { conflicto en la } \\
\text { sociedad, en la vida } \\
\text { familiar y personal. }\end{array}$ & $\begin{array}{l}\text { Conversatorio sobre los conflictos y sus } \\
\text { implicaciones. } \\
\text { Simulación de un proceso de } \\
\text { mediación, mediante un conjunto } \\
\text { de herramientas que se les dará en la } \\
\text { primera parte. }\end{array}$ \\
\hline Nov. & $\begin{array}{l}\text { Comunicación } \\
\text { asertiva }\end{array}$ & $\begin{array}{l}\text { Que los } \\
\text { participantes } \\
\text { se apropien de } \\
\text { un lenguaje } \\
\text { no violento, } \\
\text { para mejorar } \\
\text { sus procesos de } \\
\text { comunicación. }\end{array}$ & $\begin{array}{l}\text { Taller de comunicación no violenta y } \\
\text { asertiva, donde los participantes sean } \\
\text { dotados de técnicas de expresión. } \\
\text { Los participantes elaboran una muy } \\
\text { pequeña presentación oral, en la que } \\
\text { destaquen temas aprendidos a lo largo } \\
\text { de los diferentes talleres y, además, } \\
\text { pongan en práctica las herramientas e } \\
\text { insumos del taller. }\end{array}$ \\
\hline Dic. & $\begin{array}{l}\text { Actividad de } \\
\text { cierre en el CCP }\end{array}$ & $\begin{array}{l}\text { Compartir la } \\
\text { comensalidad y } \\
\text { conversar sobre los } \\
\text { aprendizajes. }\end{array}$ & Convivio de fin de año. \\
\hline
\end{tabular}

Nota: Elaborado a partir de Castillo (2016) y Angulo (2016).

Como parte de los resultados más relevantes en cuanto a percepción del contexto social, cultural y familiar, se destaca el tema sobre equidad de género; de hecho, esta temática, que no estaba programada como un taller en sí mismo, sino como eje transversal, fue solicitada por los jóvenes, cambiando la dinámica del día. Se analizó el concepto de género y los estereotipos relacionados, con sus implicaciones en la sociedad. Para las personas jóvenes, las diferencias físicas entre los sexos se han transformado en contrastes sociales, la mujer es vista como prostituta y existe un rechazo hacia la población homosexual. En relación con esto, en el taller sobre derecho a la privacidad, el grupo de jóvenes reconoce este derecho como uno de los más violentados. Plantean que los celulares han facilitado "trasiego" de fotografías que muestran partes íntimas de su cuerpo. Reconocen en su comunidad la existencia de focos de violencia y manifiestan que el sistema educativo es deficiente para abordar las situaciones descritas anteriormente, debido a que no hay campañas para el tema de la violencia ni formación en derechos humanos.

En el marco del I Festival para la Paz impulsado desde la Dirección Programática del CCP, los jóvenes elaboraron una manta titulada: "Conoce más de tus derechos y plasma tu huella", con el objetivo de percibir el conocimiento de la comunidad que asistiría a la actividad y de brindar información general de la definición de 
derechos humanos, así como de su reconocimiento. El evento mostró cómo la población no define concretamente el concepto de derechos humanos, sino que lo interpreta y ejemplifica con una acción que brinde respuesta a la carencia de un derecho en específico, se focaliza, principalmente, en el respeto, la libertad de expresión y el acceso a la educación.

Con el mapeo psicosocial comunitario participativo realizado en el marco del I Festival, los distintos sectores de la comunidad, como lo fueron niños, adolescentes, jóvenes, personas adultas y personas adultas mayores, todos ellos ubicaron su comunidad y la caracterizaron con los elementos correspondientes a recursos, fortalezas, oportunidades, debilidades, riesgos, amenazas, peligros, lugares significativos, intereses, entre otros. Destacaron como principal fortaleza la existencia del CCP de Jacó, espacio de acceso a la cultura, el deporte, el arte, la música, la tecnología y la educación; el deporte y la orientación espiritual son vistos como oportunidades; como amenazas para la comunidad, se visualizan el alcoholismo, la delincuencia y la drogadicción; adicionalmente, entre las debilidades, se hace referencia a los espacios públicos tomados por la delincuencia, el alcoholismo, la drogadicción y la prostitución. En el nivel de la escuela, determinaron el "bullying" como la principal expresión de violencia.

\section{Fortaleciendo capacidades}

Tomando como referente los resultados de la información recabada en la fase de valoración de la problemática, se determinaron y priorizaron las temáticas por atender en la siguiente fase. El proceso continuó en el primer ciclo del 2017, con 7 jóvenes del Centro Cívico para la Paz (CCP), quienes fueron parte del grupo de la valoración de la problemática, con edades entre 16 y 22 años. En esta etapa, el fortalecimiento de capacidades de liderazgo se consideraba de suma importancia, ya que se pretendía que estos jóvenes se convirtieran en promotores de derechos en su comunidad, a partir de la construcción de una propuesta de intervención en derechos humanos y cultura de paz.

En dicha fase el uso de la ludopedagogía como recurso metodológico fue fundamental, pues permitió, a través del juego, poner en práctica un amplio repertorio de actividades participativas y lúdicas con los jóvenes, las cuales facilitaron la reflexión conjunta sobre sus realidades y su entorno. Es así como reconocen que los conflictos en su comunidad se encuentran relacionados con el tema de la violencia y la resolución es mediante peleas, ataques con cuchillos o utilizando armas. El grupo consideró que el problema se daba por la intolerancia y no saber a dónde recurrir para buscar alternativas de solución. 
Los instrumentos implementados (minitareas, juego de roles) facilitaron la generación de capacidades de liderazgo en las personas jóvenes, ya que permitieron valorar las competencias desarrolladas para la resolución de conflictos, temática sobre la que se centró el proceso, porque en la etapa de valoración de la problemática, se había entretejido el fortalecimiento de capacidades en derechos humanos. En el cuadro 2 , se presenta la técnica de minitareas utilizada durante varias sesiones.

\section{Cuadro 2. Diseño de la guía de minitareas-juegos}

\begin{tabular}{|c|c|}
\hline Juegos & Minitareas \\
\hline $\begin{array}{l}\text { Periodicazo } \\
\text { Reglas del juego: El grupo está en ronda } \\
\text { y una persona deberá permanecer en el } \\
\text { centro con un periódico hecho un rollo } \\
\text { en la mano, esperando que alguno de los } \\
\text { integrantes mencione el nombre de otro del } \\
\text { mismo grupo. Al momento de pronunciarse } \\
\text { el nombre de alguien en la ronda, la persona } \\
\text { en el centro deberá, con rapidez, intentar } \\
\text { dar un periodicazo a la persona que fue } \\
\text { nombrada y esta deberá decir el nombre de } \\
\text { otro en el grupo. }\end{array}$ & $\begin{array}{l}\text { Habilidades individuales } \\
\text { Particularidades de la tarea: Este es un juego } \\
\text { en ronda, en el cual se pone en práctica las } \\
\text { habilidades de prestar atención y reaccionar } \\
\text { tanto con rapidez como correctamente, } \\
\text { cuando un mensaje trasmite una orden } \\
\text { específica. } \\
\text { Observaciones: Podemos incentivar al } \\
\text { grupo con halagos y comentarios positivos } \\
\text { sobre cómo logaron superar el desafío de } \\
\text { estar en el centro con el periódico, también, } \\
\text { repasar el nombre de las personas para } \\
\text { lograr aprendérselo y recordarlo. }\end{array}$ \\
\hline $\begin{array}{l}\text { Pasarse la cinta } \\
\text { Reglas del juego: El grupo está en ronda, } \\
\text { todos tomados de las manos; una persona } \\
\text { externa puede servir para tomar el tiempo. } \\
\text { La cinta debe pasar por el cuerpo de cada } \\
\text { persona en el grupo sin soltarse las manos; } \\
\text { puede empezar a ser pasada por la cabeza, } \\
\text { luego por el cuerpo, retirarla por los pies y } \\
\text { pasarla a la persona de la par, de esta forma, } \\
\text { hasta que la cinta pase un extremo a otro } \\
\text { del grupo. }\end{array}$ & $\begin{array}{l}\text { Cooperación en la tarea } \\
\text { Particularidades del juego: Este es un juego } \\
\text { en ronda que permite poner en marcha } \\
\text { las habilidades necesarias para alcanzar } \\
\text { objetivos, cuando el trabajo organizado sea } \\
\text { la clave para la consecución de la tarea. } \\
\text { Observaciones: Podemos incentivar al } \\
\text { grupo con mejorar el tiempo que se obtuvo } \\
\text { en el primer intento, que identifique dónde } \\
\text { se complicó el paso de la cinta y proponer } \\
\text { alguna estrategia para mejorar la trayectoria } \\
\text { de aquella. Si el grupo se acopla muy bien } \\
\text { al juego, se puede introducir una cinta más. }\end{array}$ \\
\hline
\end{tabular}




\begin{tabular}{|c|c|}
\hline Juegos & Minitareas \\
\hline $\begin{array}{l}\text { Líder, líder } \\
\text { Reglas del juego: Con el grupo en círculo, } \\
\text { comenzamos levantando un brazo y rápido } \\
\text { lo bajamos, los demás deberán hacer lo } \\
\text { mismo. Luego, se levanta un brazo y seguido } \\
\text { se levanta el otro, el grupo debe seguir los } \\
\text { movimientos y sonidos, si estos se desean } \\
\text { agregar. Los movimientos son libres y } \\
\text { pueden incluir brazos, piernas, caderas, } \\
\text { baile y posiciones corporales, los demás } \\
\text { deberán repetir lo que realice quien esté en } \\
\text { el centro del círculo. La persona del centro } \\
\text { deberá cambiar por alguien del círculo para } \\
\text { que este último sea quien integre nuevas } \\
\text { indicaciones. El juego termina cuando todos } \\
\text { los que quieran pasar al centro del círculo lo } \\
\text { puedan hacer. }\end{array}$ & $\begin{array}{l}\text { Comunicación por gestos de la persona } \\
\text { líder } \\
\text { Particularidades del juego: Es un juego } \\
\text { de ronda, la propuesta implica atención y } \\
\text { coordinación, es una dinámica para hacer } \\
\text { luego de un tiempo de proceso con el grupo. } \\
\text { La intención es trabajar la sincronización } \\
\text { grupal respecto a las direcciones provenientes } \\
\text { de la figura líder; esta puede comunicarse } \\
\text { de manera gestual, para ser replicada de la } \\
\text { misma manera por el resto del grupo. } \\
\text { Observaciones: Es un buen juego para } \\
\text { poner a prueba un líder a quien un grupo } \\
\text { sigue; así, se le permite la sensación de } \\
\text { tener a su cargo un grupo de personas que } \\
\text { están siendo orientadas y saber que, en esta } \\
\text { posición, también tiene que moverse y ceder } \\
\text { a otro el cargo de líder que dirige y orienta } \\
\text { a los demás. }\end{array}$ \\
\hline $\begin{array}{l}\text { Boliche } \\
\text { Reglas del juego: Botar el pino que se } \\
\text { colocará en el suelo a una distancia de } 5 \mathrm{~m} \text {. } \\
\text { Cada participante tendrá } 2 \text { intentos para } \\
\text { lograr botar el pino con la bola; cuando lo } \\
\text { logra, debe intentarlo nuevamente, pero } \\
\text { alejándose } 1 \mathrm{~m} \text { más. }\end{array}$ & $\begin{array}{l}\text { Habilidades individuales } \\
\text { Particularidades de la tarea: Atinar con } \\
\text { un balón al pino que está a una distancia } \\
\text { de } 5 \mathrm{~m} \text {. Es una buena oportunidad para } \\
\text { conocer las capacidades de los participantes, } \\
\text { cuando se mantienen intentando alcanzar } \\
\text { un objetivo. } \\
\text { Observaciones: Quienes participen podrán } \\
\text { tirar el balón de la forma en que se sientan } \\
\text { más cómodos, inclusive, pueden hacerlo } \\
\text { pateando el balón. }\end{array}$ \\
\hline
\end{tabular}




\begin{tabular}{|l|}
\hline Juegos \\
\hline Sin ver, ¿por quién te guiarías? \\
Reglas del juego: Un participante deberá \\
taparse los ojos con una venda para no ver \\
nada, otro esconderá el pino en un lugar \\
para ser encontrado. Luego, 2 personas del \\
grupo, en secreto, se pondrán de acuerdo \\
para darle indicaciones a quien está con los \\
ojos vendados, para que pueda encontrar \\
el pino. Una de las personas dará malas \\
indicaciones y la otra sí dará las correctas.
\end{tabular}

\section{Dogdeball países}

Reglas del juego: Cada participante escogerá un país que mientras menos sílabas tenga más conveniente le será. Luego, de todos los posicionados en una misma línea, quien tenga el balón comenzará tirándolo al aire y, en el momento en que lo haga, los demás deberán correr hacia alguna dirección. La persona que tiró el balón, mientras este está en el aire, dirá alguno de los países, para que quien escogió dicho país deba devolverse por el balón.

Cuando la nueva persona tenga el balón en sus manos, todos tendrán que quedarse en los lugares que están y aquella, con el balón en mano, dará un paso amplio por cada sílaba de alguno de los países que elija; los pasos son para acercar a la persona que representa al país y se le intentar atinar con el balón en el cuerpo. Esta última no puede moverse y, si el balón le logra tocar, quedará fuera. Gana quien no es tocado con el balón y se termina cuando expulsan a todos y solo quede una persona.

\section{Comunicación verbal en la tarea}

Particularidades del juego: Sin ver, la persona se desplaza por todo el espacio, para encontrar el pino que fue escondido. El juego invita a los participantes a decidir a qué persona hacerle caso y dejarse guiar por sus instrucciones.

Observaciones: Podemos decidir dejar de dar las indicaciones incorrectas, cuando la persona está alejándose mucho del objetivo. También, la persona que no ve estará soportando la frustración de no saber quién le está dando correctamente las indicaciones. Las personas que dan las instrucciones ponen a prueba sus habilidades de persuasión, para que quien no ve decida seguirle.

Mensaje en el canal de comunicación emisor-receptor

Particularidades del juego: Es un juego que pone a prueba la capacidad de atención y concentración para reaccionar de manera rápida y efectiva, más la toma de decisiones oportunas.

Observaciones: Es recomendable escoger un país con pocas sílabas, para que la persona con el balón no pueda acercase demasiado y le sea más difícil atinar.

Las claves están en prestar mucha atención para reaccionar con prontitud, cuando se menciona el país que le toque a cada uno, y en alejarse lo más posible, cuando el país que nos toque no es el que elegimos. 


\begin{tabular}{|c|c|}
\hline Juegos & Minitareas \\
\hline $\begin{array}{l}\text { Familia derecho e izquierdo } \\
\text { Reglas del juego: Sentados, formando un } \\
\text { círculo, quienes participen tendrán en su } \\
\text { mano algún objeto. Luego, se narrará la } \\
\text { historia que describe a una familia. Cada } \\
\text { vez que se escuche la palabra izquierda, los } \\
\text { participantes deberán pasar a la persona } \\
\text { de su izquierda el objeto que llevan en sus } \\
\text { manos; de igual manera, cuando se escuche } \\
\text { la palabra derecha, deberán pasar el objeto } \\
\text { a quien está a su derecha. El juego termina } \\
\text { cuando se puede contar la historia y fluir en } \\
\text { el traspaso de los objetos de un lado al otro. }\end{array}$ & $\begin{array}{l}\text { Cooperación y pertinencia } \\
\text { Particularidades del juego: Es un } \\
\text { juego en ronda, y la propuesta implica } \\
\text { concentración, atención y reacción. Se } \\
\text { trabaja principalmente la escucha, así como } \\
\text { la capacidad para centrarse en la tarea y } \\
\text { articularse con los demás, con el objetivo de } \\
\text { realizarla. } \\
\text { Observaciones: El juego permite sensibilizar } \\
\text { al grupo sobre el hecho de que un simple } \\
\text { cambio en una de las personas que lo } \\
\text { integran afecta, en alguna medida, al } \\
\text { colectivo en su totalidad. }\end{array}$ \\
\hline
\end{tabular}

Nota: Elaboración propia, 2019.

\section{Promotores de derechos}

Progresivamente, al finalizar la fase de "Fortalecimiento de capacidades", por razones económicas, familiares y laborales, las personas jóvenes del CCP de Jacó se desvinculan del proceso. De forma paralela, se desarrollaba en el Colegio Técnico Profesional (CTP) de Jacó un procedimiento de sensibilización para formar en derechos humanos, ejecutado por la Asociación de Estudiantes de Relaciones Internacionales y la Federación de Estudiantes de la Universidad Nacional, de abril a junio del 2017, como parte de las acciones de la Comisión del Estado de la Nación del CONARE, en el marco de la Declaratoria de Interés Institucional del año por el Diálogo, la Vida y la Paz.

Por ello, la tercera fase de esta experiencia se desarrolló como plan piloto con 24 jóvenes de 10. ${ }^{\circ}$ año del Colegio Técnico Profesional de Jacó (CTP de Jacó), quienes fueron parte de los talleres de derechos humanos mencionados y que, por lo tanto, tenían una base conceptual importante. Se tuvo la colaboración, además, de la estudiante tesiaria de la UNED, del Programa Estrechando Vínculos de la Escuela de Psicología de la UNA. Ellos aportaron la metodología del teatro espontáneo a la propuesta desarrollada en 5 sesiones y que tuvo como producto una "intervención urbana", es decir, del espacio físico del colegio.

Esa metodología permitió fomentar espacios de diálogo con las personas jóvenes, donde fueron capaces de identificar las diversas problemáticas que las aquejaban 
y, de tal manera, gracias a las herramientas provistas por cada taller, buscaron alternativas de solución (ver cuadro 3).

\section{Cuadro 3. Actividades realizadas en la fase 3 "Promotores de derechos"}

\begin{tabular}{|l|l|l|}
\hline Actividad & Objetivo/descripción & Materiales y métodos \\
\hline $\begin{array}{l}\text { Sesión 1: Diagnóstico } \\
\text { y conformación de } \\
\text { grupos, lunes 28 de } \\
\text { agosto. }\end{array}$ & $\begin{array}{l}\text { Ejercicios sociométricos: } \\
\text { Investigar características } \\
\text { grupales a través de una } \\
\text { participación activa y del uso } \\
\text { del espacio. } \\
\text { Construir el concepto de } \\
\text { promotores de paz. }\end{array}$ & $\begin{array}{l}\text { Metodología de psicodrama } \\
\text { y teatro espontáneo: En } \\
\text { subgrupos, trabajar conceptos } \\
\text { de gestores para la paz. iQué } \\
\text { son? iQue deberían hacer? iPor } \\
\text { qué son importantes? } \\
\text { Hacer una síntesis del } \\
\text { trabajo de la sesión desde lo } \\
\text { emocional. iQué se aprendió } \\
\text { en la sesión y qué podríamos } \\
\text { aportar en el proceso? }\end{array}$ \\
\hline $\begin{array}{l}\text { Sesión 2: Tema } \\
\text { "discriminación y sus } \\
\text { tipos”, viernes } 8 \text { de } \\
\text { septiembre. }\end{array}$ & $\begin{array}{l}\text { Definir el concepto de } \\
\text { discriminación desde la } \\
\text { perspectiva de los derechos } \\
\text { humanos y sus diferentes } \\
\text { manifestaciones. }\end{array}$ & $\begin{array}{l}\text { Metodología de psicodrama y } \\
\text { teatro espontáneo }\end{array}$ \\
\hline $\begin{array}{l}\text { Sesión 3: Propuestas } \\
\text { de trabajo para el tema } \\
\text { "discriminación y sus } \\
\text { tipos", jueves 21 de } \\
\text { septiembre. }\end{array}$ & $\begin{array}{l}\text { Determinar con los jóvenes } \\
\text { las intervenciones urbanas } \\
\text { participativas que elaborarán, } \\
\text { para el abordaje del derecho } \\
\text { a no ser discriminados, y sus } \\
\text { diferentes manifestaciones. }\end{array}$ & $\begin{array}{l}\text { Metodología de psicodrama y } \\
\text { teatro espontáneo }\end{array}$ \\
\hline $\begin{array}{l}\text { Sesión 4: Inicia proceso } \\
\text { de construcción de } \\
\text { intervenciones urbanas } \\
\text { participativas, lunes 2 } \\
\text { de octubre. }\end{array}$ & $\begin{array}{l}\text { En subgrupos de trabajo, } \\
\text { los jóvenes iniciarán } \\
\text { la construcción de la } \\
\text { intervención urbana } \\
\text { planteada: pinturas, murales, } \\
\text { danza, teatro, campañas, entre } \\
\text { otros. }\end{array}$ & $\begin{array}{l}\text { Sesión de exploración del } \\
\text { modelo y la experiencia } \\
\text { seleccionada. Materiales en } \\
\text { función de las intervenciones } \\
\text { urbanas planteadas. }\end{array}$ \\
\hline
\end{tabular}




\begin{tabular}{|l|l|l|}
\hline Actividad & Objetivo/descripción & Materiales y métodos \\
\hline $\begin{array}{l}\text { Sesión 5: Continuación } \\
\text { del proceso de } \\
\text { construcción de } \\
\text { intervenciones urbanas } \\
\text { participativas, jueves 19 } \\
\text { de octubre. }\end{array}$ & $\begin{array}{l}\text { Los jóvenes continuarán en } \\
\text { la construcción y finalización } \\
\text { planteada: pinturas, murales, } \\
\text { danza, teatro, campañas, entre } \\
\text { otros. }\end{array}$ & $\begin{array}{l}\text { Sesión de formulación } \\
\text { y ejecución del proceso } \\
\text { experimental de diseño } \\
\text { participativo e intervención } \\
\text { urbana. Materiales en función } \\
\text { de las intervenciones urbanas } \\
\text { planteadas. }\end{array}$ \\
\hline $\begin{array}{l}\text { Sesión 6: Ejecución } \\
\text { de las intervenciones } \\
\text { urbanas participativas, } \\
\text { miércoles 1 de } \\
\text { noviembre. }\end{array}$ & $\begin{array}{l}\text { Ejecutar las intervenciones } \\
\text { urbanas planteadas: pinturas, } \\
\text { murales, danza, teatro, } \\
\text { campañas, entre otros. }\end{array}$ & $\begin{array}{l}\text { Los jóvenes ejecutarán las } \\
\text { intervenciones en el espacio } \\
\text { público urbano de su } \\
\text { comunidad educativa, para } \\
\text { expresar su punto de vista y } \\
\text { transformar positivamente el } \\
\text { entorno. Materiales en función } \\
\text { de las intervenciones urbanas } \\
\text { planteadas. }\end{array}$ \\
\hline $\begin{array}{l}\text { Sesión 7: Planificación } \\
\text { de trabajo 2018, } \\
\text { miércoles } 8 \text { de } \\
\text { noviembre. }\end{array}$ & $\begin{array}{l}\text { Llevar a cabo una sesión de } \\
\text { retroalimentación con los } \\
\text { jóvenes, sobre la experiencia } \\
\text { de las intervenciones urbanas } \\
\text { realizadas. }\end{array}$ & $\begin{array}{l}\text { Realizar un proceso evaluativo } \\
\text { con los jóvenes de 10. año } \\
\text { del CTP de Jacó y construir } \\
\text { una propuesta de trabajo para } \\
\text { el 2018, que trascienda su } \\
\text { comunidad educativa. }\end{array}$ \\
\hline
\end{tabular}

Nota: Elaboración propia, 2019.

Se analizaron el patriarcado, las relaciones de poder y las construcciones jerárquicas de la masculinidad y la feminidad, como los motores que generan la violencia de género en sus distintas expresiones. Lo anterior brindó sustento para que los jóvenes del CTP de Jacó identificaran como problemática principal el tema del sexting y la violencia de género, la cual podría ser abordada mediante el desarrollo de teatro, foros y videos, técnicas más atractivas y lúdicas para mostrar las consecuencias de la problemática a la población estudiantil del CTP y a otras personas jóvenes de la comunidad de Jacó.

Al final de la experiencia, las personas participantes se identificaron como futuros promotores de derechos en el colegio, de hecho, iniciaron coordinaciones para orientar un proceso similar el año siguiente, con estudiantes que realizarán el trabajo comunitario. La institución se mostró anuente a continuar con la experiencia. 


\section{Desde nuestras lecciones aprendidas}

Educar para una cultura de paz significa educar para la crítica y la responsabilidad, para la comprensión y el manejo positivo de los conflictos, así como potenciar los valores del diálogo y el intercambio y revalorizar la práctica del cuidado y de la ternura, todo ello como una educación pro-social que ayude a superar las dinámicas destructivas y a enfrentarse a las injusticias.

Viçen Fisas Armengol

Desde la experiencia desarrollada con los jóvenes del cantón de Garabito, se establece una serie de recomendaciones para la ejecución de una propuesta como esta.

\section{Conocer la dinámica sociocultural de los jóvenes y sus familias}

En procesos como estos, el conocer el entorno en que viven los jóvenes (rural), la estructura familiar, el nivel socioeconómico, el de escolaridad, así como los niveles de violencia en la comunidad es fundamental, pues podrían representar una dificultad para el ejercicio del liderazgo cívico por parte de los participantes.

Vásquez y Mansilla (2005) señalan que los desniveles educativos en los jóvenes podrían limitar, o bien hacer más largo el proceso de desarrollo de habilidades como el liderazgo, debido a la poca capacidad comunicativa de los jóvenes producto de su pobreza lingüística y académica, en temas conceptuales de todo tipo. En su quehacer, ellos encontraron mayores dificultades para propiciar trabajos en grupo con responsabilidades compartidas, más bien, se daban acciones buscando el beneficio individual, con muy poca formalidad en la presentación de las tareas, así como la falta de puntualidad. En nuestra experiencia, en especial con el grupo de jóvenes del CCP de Jacó, debido a su irregularidad en la asistencia a los talleres, fue como trabajar con un grupo nuevo en cada sesión de trabajo convocada.

El tema familiar es otro punto por considerar, pues podría influir en los niveles de participación y compromiso; responde diferente un muchacho quien procede de un hogar con experiencia y tradición familiar en participación comunitaria, que aquel cuya familia se opone o no le interesa una acción de formación y participación ciudadana. Por lo tanto, los líderes que mejor desarrollan sus capacidades sociales son aquellos que tienen referentes familiares de participación o familias que apoyan y promocionan la participación ciudadana (Vásquez y Mansilla, 2005).

Las situaciones de conflicto y violencia en el nivel comunitario también deben ser reconocidas, por lo que resulta necesario considerar actividades que desarrollen 
fortalezas de resolución, conciliación y mediación en todos los ámbitos (familia, pareja, vecinos, colegio, trabajo), desde los espacios formales (centros educativos) y los informales.

\section{El rol que debe cumplir el promotor}

Al plantearse una propuesta de trabajo con jóvenes como promotores de derechos y paz, es importante considerar un proceso de formación teórico-práctica, con la cual se explique el rol del promotor, el cual viene siendo el de atender las inquietudes, preguntas e iniciativas de los demás jóvenes. Si bien es cierto debe conocer aspectos teóricos, su función no es formar, es atender y motivar al grupo para con ello logar la permanencia y la perseverancia de los jóvenes, así como el desarrollo de actividades grupales. Su función va más allá del acompañar o guiar al grupo (ayudando a que se organice o a que aplique lo aprendido): es la persona de confianza, que comparte con ellos los espacios de acción cívica, pero no quien los ejecuta.

Algo fundamental en este aspecto es apoyar a los jóvenes promotores con capacitaciones en metodologías lúdicas, para hacer del aprendizaje algo divertido y con buena participación. Cerdas (2013) sostiene que los juegos cooperativos generan oportunidades vivenciales y divertidas para el aprendizaje conjunto, cooperativo, creativo y respetuoso, en el cual se reconocen y aprecian las diferencias, porque permiten que se refuerce la autoconfianza, como personas dignas y de valor.

En este sentido, entonces, otro aspecto importante por considerar es que la propuesta pedagógica planteada agrupe, de forma permanente, la teoría y la práctica, el pensamiento y el juego bajo las mismas actividades.

\section{La ludopedagogía como ente articulador en el proceso formativo}

Considerando que durante la experiencia se tuvo problemas de presencia y permanencia de participantes jóvenes, en especial los del CCP de Jacó, quisiéramos recalcar la importancia de la ludopedagogía como experiencia de aprendizaje a través del juego. Lo vivido expone, una vez más, cómo el trabajar desde lo lúdico acerca a los jóvenes y los estimula a interrelacionarse con otros de su edad.

El impacto de la metodología en los participantes se aprecia cuando estos desarrollan un reconocimiento de la identidad tanto individual como colectiva, valoran las diferencias, el aprendizaje cooperativo, la expresión de sentimientos y opiniones, entre otros. Es importante destacar que dicha metodología puede involucrar 
a otros sectores sociales (familia y comunidad) que forman parte del entorno de la población juvenil, para con ello establecer acciones conducentes a un modelo de convivencia y resolución de conflictos integral. Las sesiones de trabajo bajo esta herramienta formativa propician espacios en los cuales pueden expresar lo que sienten, mejorar sus relaciones interpersonales y valorar sus capacidades, para reconocerse como agentes multiplicadores de su experiencia de formación en derechos humanos y cultura de paz.

\section{¿Habrá deserción?}

A pesar de que el trabajar con jóvenes implica algún nivel de deserción, lo que pasó en esta experiencia, en especial con los muchachos del CCP, quisiéramos exponer algunas de las razones que motivaron la movilidad en los jóvenes, como reflexión para trabajos similares.

La radiografía del contexto sociocultural en este tipo de vivencias es fundamental, debido a que deja en evidencia que las personas jóvenes experimentan situaciones significativas de violencia en el seno familiar, las cuales marcan sus vidas, interviniendo en el entretejido cultural, comunitario e individual. Entre las más importantes, figura la edad, seguida de los compromisos de estudio, como cambios de trabajo (despidos). Vásquez y Mansilla (2005) señalan que "la elección de trabajar con un estrato social medio y bajo complica la permanencia". En su experiencia de trabajo "muchos de los jóvenes se retiraron del proyecto porque migraron a otras zonas a buscar trabajos, porque la situación económica de sus familias obligaba a que den sus tiempos libres para buscar otro tipo de trabajos, etc." (p. 143). Todas estas circunstancias deben considerarse como un riesgo al momento de trabajar con los jóvenes, pues a muchos, aunque puedan manifestar interés, les terminan pesando más los aspectos familiares y económicos.

\section{Referencias}

Angulo, P. (2016). Caracterización de la ciudad de Jaco y formación de jóvenes líderes en el Centro Cívico para la Paz de Garabito. (Informe de Práctica Profesional Supervisada para culminar el nivel de Bachillerato en Psicología. En el curso Práctica Profesional Supervisada. Escuela de Psicología, Universidad Nacional, Heredia).

Barahona, F. (2008). Educando para una cultura de paz. San José, Costa Rica: EUNED.

Barquero-Brenes, R. (2014). Convivencia en el contexto familiar: un aprendizaje para construir cultura de paz. Revista Electrónica Actualidades Investigativas en Educación, 14(1), 1-19. Recuperado de file:///F:/ARTICULO\%20IDELA/Tesis\%202017\%20UCR\%20Cultura\%20 de\%20paz.pdf 
Castillo, J. (2016). Derechos Humanos y Cultura de Paz. (Informe de Práctica Profesional Supervisada para culminar el nivel de Bachillerato en Relaciones Internacionales. En el curso Práctica Profesional Supervisada. Escuela de Relaciones Internacionales, Universidad Nacional, Heredia).

Chan, J.; Sánchez, N. y Víquez, C. (2017). Viviendo la violencia: percepción de niños. niñas y adolescentes en zonas de riesgo social del cantón de Garabito, Puntarenas, Costa Rica. Trama, revista de ciencias sociales y humanidades, 6(2), 13-24.

Cerdas-Agüero, E. (2013). Experiencias y aprendizajes con juegos cooperativos. Revista Paz y conflictos, 6, 107-123. Recuperado de https://www.google.com/search?q=Experiencias+ ${ }^{+}$aprendizajes ${ }^{+}$con + juegos ${ }^{+}$cooperativos $\&$ oq $=$ Experiencias ${ }^{+}{ }^{+}$aprendizajes ${ }^{+}$con + juegos ${ }^{+}$cooperativos\&aqs=chrome. $.69 \mathrm{i} 57.1250 \mathrm{j} 0 \mathrm{j} 8 \&$ sourceid $=$ chrome\& $\mathrm{ie}=\mathrm{UTF}-8$

Fondo de las Naciones Unidas para la Infancia (UNICEF). (2006). Cuadernillo 3: Participación de niños, niñas y adolescentes. Argentina: UNICEF. Recuperado de https://studylib.es/ doc/4824577/participaci\%C3\%B3n-de-ni\%C3\%B1os-ni\%C3\%B1as-y-adolescentes

Fulladosa, R. (2015). Con-jugando aportes: entre el juego y la pedagogía. Ludopedagogía y Trabajo Social. San José, Costa Rica: UCR. Recuperado de https://www.colibri.udelar.edu.uy/jspui/ bitstream/20.500.12008/18710/1/TTS_FulladosaTania.pdf

Garrido, R. (17 de enero de 2017). La paz requiere sociedades que vivan en equidad, libertad, igualdad y solidaridad. Barranquila, Colombia: Fundación Teknos. Recuperado de https:// fundacionteknos.org/cultura-de-paz-y-derchos-humanos/

Grillo, M. (2014). Modelo base de atención Centros Cívicos por la Paz (CCP). (Trabajo de consultoría). San José, Costa Rica: Ministerio de Justicia y Paz.

Las Naciones Unidas. (1948). Declaración Universal de Derechos Humanos. Recuperado de https://www.un.org/chinese/center/chbus/events/hurights/spanish.htm/

Organización de las Naciones Unidas para la Educación, la Ciencia y la Cultura (UNESCO). (1997). Contribución del Director General al informe del Secretario General de las Naciones Unidas sobre el proyecto transdisciplinario "Hacia una cultura de paz". Recuperado de https://unesdoc.unesco.org/ark:/48223/pf0000108719_spa/PDF/108719spa.pdf.multi

Rickly, W. y Ara del Amo, O. (2011). ¿Qué es la ludopedagogía? Enfoque y metodología. (Propuesta elaborada en el marco del curso Gestionar jugando: experimentación metodológica en cultura). España: Universidad Internacional de Andalucía (UNIA). Recuperado de http:// www.zemos98.org/eduex/IMG/pdf/QUE_ES_LA_LUDOPEDAGOGIA.pdf

Trucco, D. e Inostroza, P. (2017). Las violencias en el espacio escolar. Chile: CEPAL . Recuperado de https://repositorio.cepal.org/bitstream/handle/11362/41068/4/S1700122_es.pdf 
UNESCO. (2008). Convivencia democrática, inclusión y cultura de paz. Santiago, Chile: UNESCO. Recuperado de https://unesdoc.unesco.org/ark:/48223/pf0000162184

Universidad Nacional, Vicerrectoría de Extensión. (2016). Formulación del proyecto: Ciudades Culturales: UNA Alianza para el Futuro. Código SIA 0598-15.

Vásquez-Bermejo, O. y Mansilla-Torres, K. (2005). Construyendo país. Experiencias de Participación Ciudadana promovida por jóvenes de Piura, Lima, Moquegua, Trujillo y Cusco. (Consorcio Juventud y País). Recuperado de http://centroderecursos.alboan.org/sistematizacion/ es/registros/1826-construyendo-pais-experiencias-de 